\title{
Susceptibility to Peer Pressure as an Explanatory Variable for the Differential Effectiveness of an Alcohol Misuse Prevention Program in Elementary Schools
}

\author{
T.E. Dielman, Deborah D. Kloska, Sharon L. Leech, \\ John E. Schulenberg, Jean T. Shope
}

\begin{abstract}
A school-based alcohol misuse prevention program had differential effects on students' susceptibility to peer pressure, depending on prior experience with alcohol. These effects paralleled those on alcohol use and misuse, indicating program effects on use and misuse were mediated by reductions in the rate of increase on susceptibility to peer pressure. Experimental group students with prior unsupervised use of alcohol showed a significantly greater reduction than their controls in the rate of increase in susceptibility to peer pressure, alcohol use, and alcohol misuse. This difference was not found among students without prior unsupervised use of alcohol. (J Sch Health. 1992;62(6):233-237)
\end{abstract}

$\mathbf{G}^{\circ}$ oodstat' noted that most previous substance abuse prevention studies evaluated the prevention programs without regard to the possibility that prior abstainers may differ from prior users in their response to the prevention program. Combining these two groups in the program evaluation could attenuate or completely mask a program effect. This masking could be detected if the students' experience with the substance at pretest were included as a stratification variable in the analysis. Dielman et $\mathrm{al}^{2}$ found a reduction in the rate of increase of alcohol use and misuse (AUM) among experimental group students who had experienced unsupervised use of alcohol prior to the intervention. Students who were abstainers or who had experienced only adult supervised alcohol use prior to the intervention exhibited low AUM rates at all testing occasions, with no differential rates of increase between experimental and control groups. In a subsequent report, Dielman et $\mathrm{al}^{3}$ found that adolescents' level of susceptibility to peer pressure (SPP) had the most salient direct effect on AUM in a structural equation model that also included grade level, exposure to peer use and misuse of alcohol, internal health locus of control, and self-esteem as predictors. This study tested the differential effectiveness of the intervention with respect to rate of increase in adolescents' SPP, and the consequent effect of the rate of SPP increase on the rate of AUM increase.

T.E. Dielman, PhD, Professor; Deborah D. Kloska, BS, Research Associate: Sharon L. Leech, MPH, Research Associate; and Jean $T$. Shope, PhD, Assistant Professor, Dept. of Postgraduate Medicine and Health Professions Education, University of Michigan, G1200 Towsley Center, Ann Arbor, MI 48109-0201; and John E. Schulenberg, PhD, Assistant Research Scientist, 2030 Institute for Social Research, Box 1248, 426 Thompson St., Ann Arbor, MI 48106-1248. This research was supported by grant no. AA06324 from the National Institute on Alcohol Abuse and Alcoholism. This article was submitted November 18, 1991, and revised and accepted for publication March 16, 1992.

\section{METHOD}

The Alcohol Misuse Prevention Study (AMPS), being conducted by the authors, is based on a social skills/ peer pressure resistance approach and addresses many of the methodological shortcomings noted in earlier studies. A conceptual model specifying the hypothesized direct and indirect program effects on intervening and outcome variables guided development of the AMPS program objectives, curriculum materials, and evaluation instruction. ${ }^{2}$ The design and implementation of the study and the curriculum have been described elsewhere. ${ }^{2-10} \mathrm{~A}$ randomized, pre-post, experimental-control design, following individual students longitudinally, was used to evalute the program. Students from 49 schools were assigned randomly to conditions by school building. All students were post-tested at the end of the first school year after program implementation, as well as at the end of the two subsequent years.

Care was taken during the implementation and evaluation phases to ensure that any differences found between experimental and control groups could be attributed to effects of the prevention program. Schools were matched on socioeconomic, ethnic, and achievement variables prior to random assignment to treatment conditions. A description of the data management and analysis procedures is provided elsewhere. ${ }^{2,4}$ The analyses conducted for this report were based on 714 sixth grade students who were pretested and who were present at all four testing occasions. Questionnaire items included in these analyses measured quantity and frequency of alcohol use, misuse of alcohol (overindulgence, trouble with peers, and trouble with adults), SPP, and type of prior experience with alcohol. The item content, scoring and index construction procedures, score ranges, and alpha coefficients for the variables used in the current analyses have been described previously. ${ }^{24,10}$ In the first set of analyses, the dependent 
variable was SPP, with treatment condition, occasion, and type of prior drinking experience as independent variables. In subsequent analyses, the total alcohol misuse and alcohol frequency-quantity scores were the dependent variables, with treatment condition, occasion, type of prior drinking experience, and level of SPP (low/medium and high) as independent variables.

The primary hypothesis of interest in this study held that the difference between the experimental and control groups in the rate of SPP increase over time would differ depending on type of prior drinking experience. This view is expressed by hypothesizing a significant threeway (treatment condition by type of prior drinking experience by occasion) interaction, indicating the program had differential treatment effects over time, depending on the student's type of prior drinking experience. A three-way repeated measures analysis of variance (treatment condition by type of prior drinking experience by occasion), weighted for unequal cell sizes, was conducted, using SPP as the dependent variable. Independent variables were the students' type of prior drinking experience (three levels), treatment (two levels), and testing occasion (four levels). Preliminary one-way analyses of variance of the pretest SPP scores indicated no significant differences among group means and variances at the pretest occasion. The repeated measures analyses of variance were conducted using the SPSS-X subroutine designed for that purpose. "When the analysis resulted in a significant F-ratio for a main or interaction effect with more than two levels, comparisons among pairs of group means were conducted using Scheffe tests, which provide a conservative test of between group differences when making multiple $a$ posteriori comparisons.

In the experimental design, the unit of randomization was the school rather than the individual student. The analysis, however, used the individual student as the unit of analysis for two reasons: 1) the need to track individual students over time to assess individual behavior, and 2) the fact that students did not move to their new grade levels in intact classes. Use of different units in assignment to conditions and for analytic purposes presented a methodological problem in that the alcohol use and misuse behavior of students within schools tends to be correlated and cannot be considered independent. ${ }^{2,8,12-14}$ Suggested analytic strategies for compensating for this situation have been specification of the grouping factor in hierarchical analysis of variance or use of group means as the unit of analysis. 2,8,14,15 These approaches are not useful in longitudinal studies in which groups do not remain the same over time and in which there is a desire to track longitudinally the behavior of individuals who are exposed to different experimental conditions. Another applicable technique, suggested by the survey sampling literature ${ }^{16}$ and, more recently, by school-based prevention researchers, 14 has been employed by the authors previously, 2,8 as well as in the current analyses, to adjust for the effect of clustering of students within schools. With this approach, new sampling variances are calculated which take clustering into account. To the extent that students within schools resemble each other on dependent variables, as measured by the intraclass correlations for those variables, the new sampling variances will be larger than those which ignore the clustering. In the current analyses, variances corrected for clustering were calculated when significant effects were found.

As a final step to test the hypothesis that SPP served as a mediating variable with respect to the effect of the intervention on AUM, structural equation analyses were conducted within each of the type of prior drinking experience subgroups. If SPP serves as a mediating variable, then the indirect effect of the intervention, through SPP, on changes in AUM from pretest to the final posttest should be significant, especially within the subgroup with prior unsupervised drinking experience.

\section{RESULTS}

The cell sizes, means, and standard deviations by treatment condition, type of prior drinking experience, and occasion are presented in Table 1 for SPP. The re-

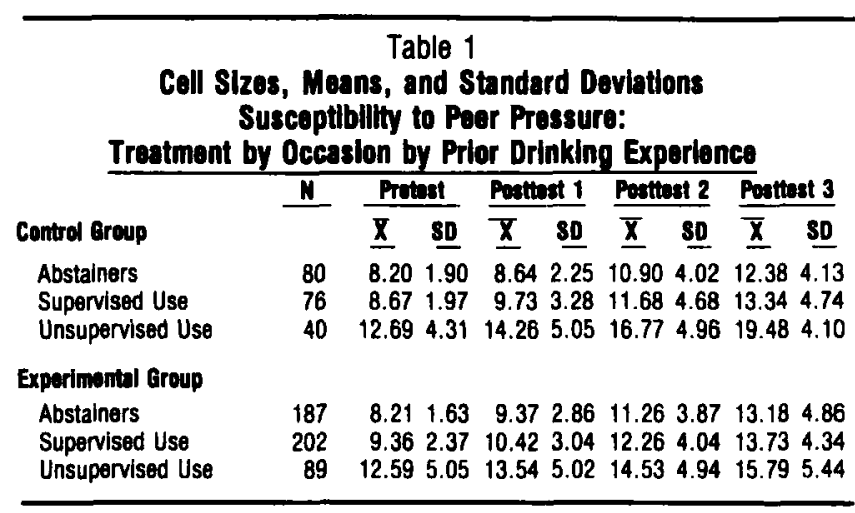

Figure 1

Suscoptiblilty to Poer Pressuro:

Treatment by Occasion by Prior Drinking Experience

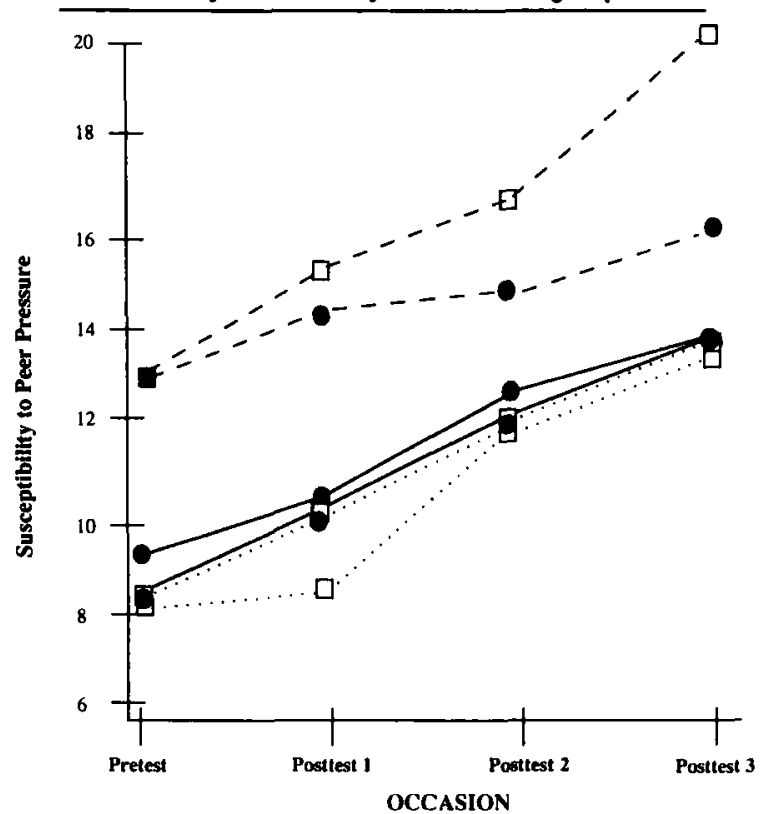

OCCASION

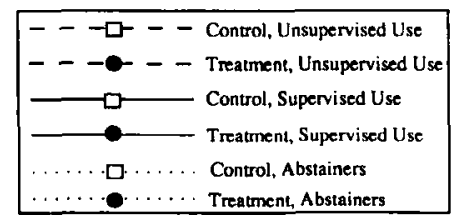


$(p<.001)$ for the main effect of type of prior drinking experience, $51.3(p<.001)$ for the main effect of SPP, $61.3(p<.001)$ for the main effect of testing occasion, $3.6(p<.06)$ for the interaction of treatment condition by SPP, and $4.8(p<.01)$ for the interaction of type of prior drinking experience by SPP.

The repeated measures analysis of variance of the total alcohol misuse scores resulted in significant main effects of testing occasion $(\mathrm{F}=80.8, p<.001, \mathrm{df}=$ $3 / 1,929)$, type of prior drinking experience $(F=56.8$, $p<.001, \mathrm{df}=2 / 643)$, and SPP $(\mathrm{F}=82.8, p<.001$, $\mathrm{df}=1 / 643$ ). The intraclass correlation coefficient for total alcohol misuse was .006 , yielding a design effect of 1.15. When corrected for the design effect, the F-ratios were $70.3(p<.001)$ for testing occasion, 49.4 $(p<.001)$ for type of prior drinking experience, and $72.0(p<.001)$ for SPP. The main effect of treatment condition was not significant. Significant two-way interactions were found for type of prior drinking experience by SPP $(F=5.2, p<.01$, df $=2 / 643$; corrected for design effect, $F=4.5, p<.01$ ), treatment condition by testing occasion $(F=3.7$, $p<.01, \mathrm{df}=3 / 1,929$; corrected for design effect, $\mathrm{F}=$ $3.2, p<.02)$, and SPP by testing occasion ( $\mathrm{F}=36.7$, $p<.001, \mathrm{df}=3 / 1,929$, corrected for design effect, $F$ $=31.9, p<.001$ ). There was a marginal two-way interaction of treatment condition by SPP (F $=2.9, p=$ $.09, \mathrm{df}=1 / 643$ ). Treatment condition by type of prior drinking experience and type of prior drinking experience by testing occasion were not significant. The uncorrected three-way interaction of type of prior drinking experience by SPP by testing occasion was

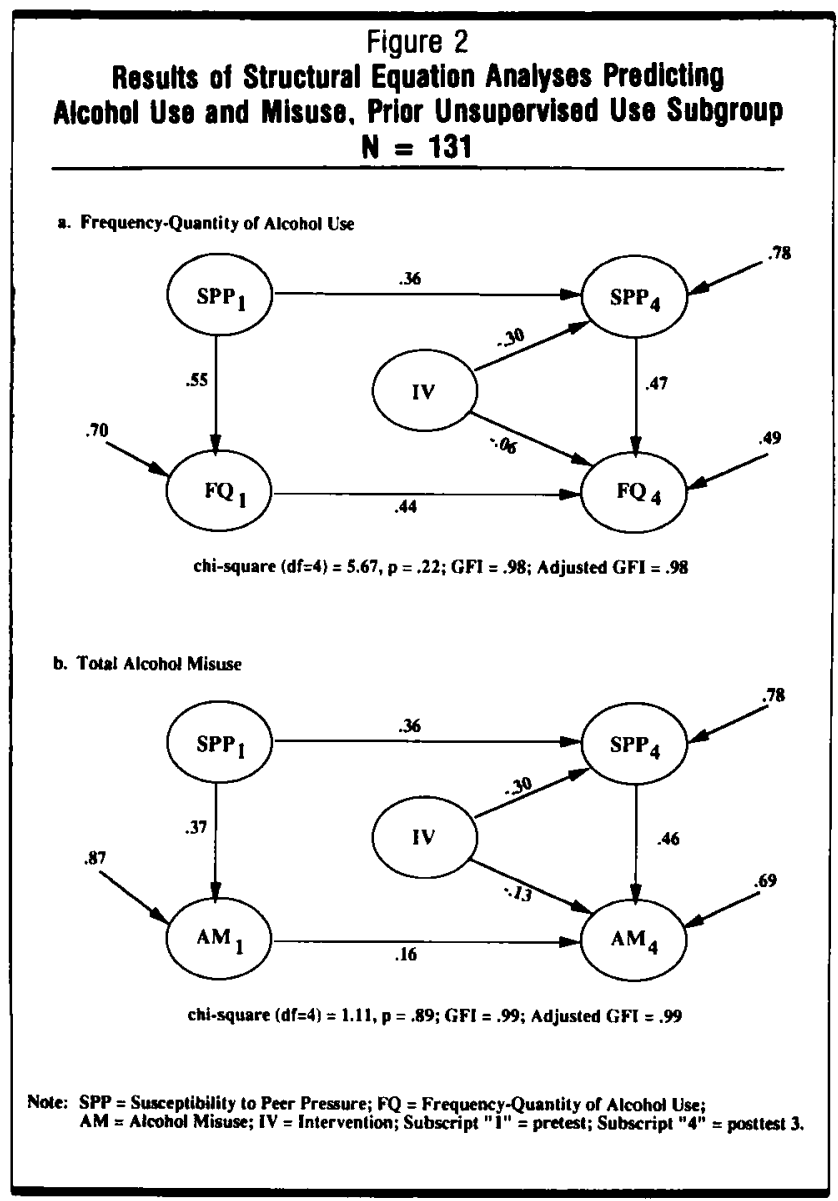

significant $(\mathrm{F}=2.3, p<.04, \mathrm{df}=6 / 1,929$; corrected for design effect, $\mathrm{F}=2.0, p<.07)$. There were no other significant effects.

The third approach to test the mediating effects of SPP was to conduct two series of structural equation analyses. Results of structural equation analyses for the sixth grade students with prior unsupervised drinking experience are shown in Figure 2 for both the frequencyquantity of alcohol use and total alcohol misuse. As shown in Figure 2, the stability coefficients for SPP and the frequency-quantity of alcohol use were .36 and .44 , respectively, from pretest to posttest three. The coefficient relating SPP to the frequency-quantity of alcohol use at pretest was .55. The coefficient for the effect of SPP on the change in the frequency-quantity of alcohol use was .47. The direct effect of the intervention on the change in SPP was -.30, and the direct effect of the intervention on change in the frequency-quantity of alcohol use was -.06 . When the effect of the intervention on change in SPP was taken into account, the indirect effect of the intervention on the frequencyquantity of alcohol use through the change in SPP was -.14 , and the remaining direct effect of the intervention on the frequency-quantity of alcohol use was -.06 . The total effect of the intervention on the students' frequency-quantity of alcohol use thus was -.20 .

As shown in Figure 2, the stability coefficient for total alcohol misuse was .16 and the coefficient relating SPP to total alcohol misuse at pretest was .37. The coefficient for the direct effect of SPP on the change in total alcohol misuse was .46. The direct effect of the intervention on change in total alcohol misuse was -.13. Controlling for the effect of the intervention on SPP, the indirect effect of the intervention on total alcohol misuse through SPP was -.14, and the remaining direct effect of the intervention on total alcohol misuse was -.13 . The total effect of the intervention on total alcohol misuse consequently was -.27 .

\section{DISCUSSION}

Results from this study indicate the school-based resistance to social pressure skills prevention program reduced the rate of increase in adolescents' SPP, and this reduction was directly related to reduced rates of increase in adolescent AUM. These findings support the hypothesis that the differential effectiveness of this approach to the prevention of adolescent AUM is mediated to a great extent by changes in the students' SPP. The implication for prevention program development is that the social pressure resistance skills approach used in this study should continue to be a focus in school-based substance abuse prevention programs. A limitation of the study is the small number of students $(N=131)$ in the subgroup for whom the prevention program was most effective. A further test of the causal model with a larger sample is necessary.

The finding that this mediated effect occurred among students who had begun to experiment with alcohol use in unsupervised settings prior to the intervention, and that this subgroup had initially higher rates of AUM, as well as higher initial SPP scores, suggests the possibility that earlier intervention might be effective in preventing such early experimentation. This 
peated measures analysis of variance resulted in a significant main effect of occasion $(F=266, p<.001)$, and significant interaction effects of treatment by occasion $(F=4.1, p<.01)$ and treatment by type of prior drinking experience by occasion ( $F=4.1, p<.001)$. The intraclass correlation for SPP was .004, yielding a design effect of 1.10. When corrected for the design effect, the F-ratios were 242 for occasion ( $\mathrm{df}=$ $3 / 2,004 ; p<001$ ), 3.8 for treatment by occasion ( $\mathrm{df}=$ $3 / 2,004 ; p<.05$ ), and 3.8 for treatment by type of prior drinking experience by occasion ( $\mathrm{df}=6 / 2,004$; $p<.001)$.

Mean SPP scores are plotted by testing occasion in Figure 1 for each treatment group by type of prior drinking experience subgroup. Table 1 and Figure 1 indicate SPP means were lowest at pretest for the abstainer group, somewhat higher for the supervised

Table 2

Cell Sizes, Means, and Standard Deviations Frequency-Quantity of Alcohol Use Index by Treatment Condltion, Type of Prior Drinking Experionce, and Susceptiblity to Poer Pressure (low-modlum vs. high)

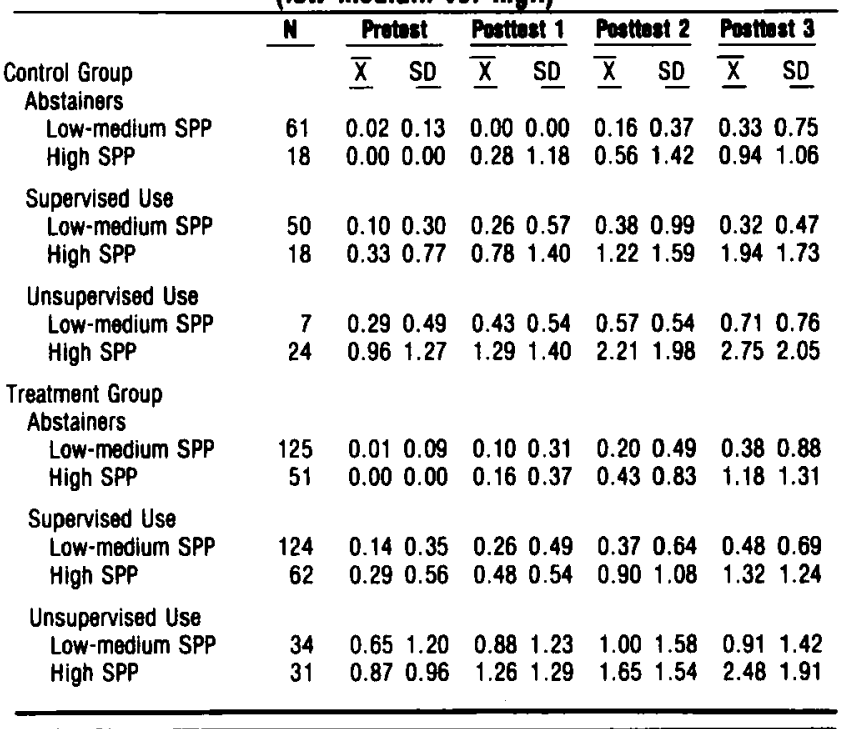

Table 3

Cell Sizes, Means, and Standard Dovlations

Total Alcohol Misuse Index by Treatment Condtion, Type of Prior Drinking Experience, and Susceptibility to Peer Pressure (low-medium vs. high)

\begin{tabular}{|c|c|c|c|c|c|c|c|c|}
\hline \multirow[b]{2}{*}{$\begin{array}{c}\text { Contral Group } \\
\text { Abstainers }\end{array}$} & \multirow[t]{2}{*}{ N } & Protost & \multicolumn{2}{|c|}{ Posttost 1} & \multicolumn{2}{|c|}{ Posttest 2} & \multicolumn{2}{|c|}{ Pusttest 3} \\
\hline & & $\underline{\bar{x}} \quad \underline{S D}$ & $\underline{\bar{x}}$ & SD & $\underline{\bar{x}}$ & $\underline{S D}$ & $\underline{x}$ & SD \\
\hline $\begin{array}{l}\text { Low-medium SPP } \\
\text { High SPP }\end{array}$ & $\begin{array}{l}62 \\
19\end{array}$ & $\begin{array}{ll}0.03 & 0.18 \\
0.00 & 0.00\end{array}$ & $\begin{array}{l}0.08 \\
0.47\end{array}$ & $\begin{array}{l}0.33 \\
1.22\end{array}$ & $\begin{array}{l}0.27 \\
0.53\end{array}$ & $\begin{array}{l}0.61 \\
1.07\end{array}$ & $\begin{array}{l}0.31 \\
1.63\end{array}$ & $\begin{array}{l}0.80 \\
1.46\end{array}$ \\
\hline $\begin{array}{l}\text { Supervised Use } \\
\text { Low-medium SPP } \\
\text { High SPP }\end{array}$ & $\begin{array}{l}53 \\
23\end{array}$ & $\begin{array}{ll}0.25 & 0.55 \\
0.39 & 0.66\end{array}$ & $\begin{array}{l}0.34 \\
0.65\end{array}$ & $\begin{array}{l}0.76 \\
1.23\end{array}$ & $\begin{array}{l}0.36 \\
1.04\end{array}$ & $\begin{array}{l}0.81 \\
1.33\end{array}$ & $\begin{array}{l}0.43 \\
2.48\end{array}$ & $\begin{array}{l}0.91 \\
1.93\end{array}$ \\
\hline $\begin{array}{l}\text { Unsupervised Use } \\
\text { Low-medium SPP } \\
\text { High SPP }\end{array}$ & $\begin{array}{r}7 \\
32\end{array}$ & $\begin{array}{ll}1.14 & 1.22 \\
1.50 & 1.52\end{array}$ & $\begin{array}{l}0.14 \\
1.57\end{array}$ & $\begin{array}{l}0.38 \\
1.63\end{array}$ & $\begin{array}{l}0.43 \\
2.06\end{array}$ & $\begin{array}{l}0.54 \\
1.80\end{array}$ & $\begin{array}{l}1.71 \\
3.25\end{array}$ & $\begin{array}{l}2.43 \\
1.67\end{array}$ \\
\hline $\begin{array}{l}\text { Treatmont Group } \\
\text { Abstainers }\end{array}$ & & & & & & & & \\
\hline $\begin{array}{l}\text { Low-medium SPP } \\
\text { High SPP }\end{array}$ & $\begin{array}{r}122 \\
57\end{array}$ & $\begin{array}{ll}0.03 & 0.22 \\
0.00 & 0.00\end{array}$ & $\begin{array}{l}0.16 \\
0.16\end{array}$ & $\begin{array}{l}0.60 \\
0.49\end{array}$ & $\begin{array}{l}0.30 \\
0.61\end{array}$ & $\begin{array}{l}0.85 \\
1.16\end{array}$ & $\begin{array}{l}0.31 \\
1.32\end{array}$ & $\begin{array}{l}0.92 \\
1.50\end{array}$ \\
\hline $\begin{array}{l}\text { Supervised Use } \\
\text { Low-medium SPP } \\
\text { High SPP }\end{array}$ & $\begin{array}{r}129 \\
65\end{array}$ & $\begin{array}{ll}0.21 & 0.46 \\
0.40 & 0.63\end{array}$ & $\begin{array}{l}0.31 \\
0.42\end{array}$ & $\begin{array}{l}0.83 \\
0.68\end{array}$ & $\begin{array}{l}0.34 \\
0.72\end{array}$ & $\begin{array}{l}0.76 \\
1.24\end{array}$ & $\begin{array}{l}0.61 \\
1.91\end{array}$ & $\begin{array}{l}1.28 \\
1.88\end{array}$ \\
\hline $\begin{array}{l}\text { Unsupervised Use } \\
\text { Low-medium SPP } \\
\text { High SPP }\end{array}$ & $\begin{array}{l}42 \\
44\end{array}$ & $\begin{array}{ll}1.21 & 1.69 \\
1.25 & 1.74\end{array}$ & $\begin{array}{l}1.00 \\
1.61\end{array}$ & $\begin{array}{l}1.38 \\
1.74\end{array}$ & $\begin{array}{r}.98 \\
2.18\end{array}$ & $\begin{array}{l}1.26 \\
1.97\end{array}$ & $\begin{array}{l}1.00 \\
2.75\end{array}$ & $\begin{array}{l}1.29 \\
2.14\end{array}$ \\
\hline
\end{tabular}

drinking experience group, and significantly higher than the rest for the unsupervised drinking experience group. Pretest means for the experimental and control groups within types of prior drinking experience classifications did not differ significantly. Rates of increase in SPP means across occasions were nearly identical for the experimental and control groups within the abstainer and supervised experience groups. In the prior unsupervised experience subgroup, however, the rate of increase of SPP means across occasions was significantly greater for the control group than for the experimental group. The difference between the experimental and control group for the unsupervised prior experience students was statistically significant at the posttest three occasion $(\mathrm{t}=3.0, p<.01 ;$ corrected for design effect $\mathrm{t}=2.7$, $p<.01)$, but not at the posttest two occasion $(t=1.7)$.

These results of the analyses of the SPP scores by treatment condition, type of prior drinking experience, and occasion paralleled the analyses of the rates of increase in the students' alcohol use and misuse reported by Dielman et al. ${ }^{2}$ This finding suggests that the effects of the alcohol misuse prevention program were achieved in large part through a reduction in the students' SPP. Three additional sets of analyses were conducted to confirm this possibility. First, the students' pretest to posttest three SPP change scores were correlated with their change scores over the same testing occasions on frequency-quantity of alcohol use and total alcohol misuse. The correlation of the change on SPP was .45 with the change on the frequency-quantity of alcohol use $(p<.001)$, and .39 with the change on the total alcohol misuse $(p<.001)$. The correlation of change in the frequency-quantity of alcohol use with the change in the total alcohol misuse was $.57(p<.001)$.

Second, repeated measures analyses of variance on the frequency-quantity of alcohol use and total alcohol misuse were conducted using treatment condition, type of prior drinking experience at pretest, posttest three SPP, and occasion as independent variables. The cell sizes, means, and standard deviations for the repeated measures analyses of frequency-quantity of alcohol use and total alcohol misuse are presented in Tables 2 and 3, respectively. The SPP scores at posttest three were grouped into two categories: low/medium (scores of 7 through 15.75, $\mathrm{N}=447$ ) and high (scores of 16-28, $\mathrm{N}=$ 257). This grouping was done on the combined basis of the meaning and the distribution of scores.

The repeated measures analysis of variance of the alcohol frequency-quantity scores resulted in significant main effects of type of prior drinking experience $(\mathrm{F}=$ $45.4, p<.001, \mathrm{df}=2 / 593), \operatorname{SPP}(\mathrm{F}=82.1, p<.001$, $\mathrm{df}=1 / 593)$, and testing occasion ( $\mathrm{F}=98.1, p<.001$, $\mathrm{df}=3 / 1,799)$. The main effect of treatment condition was not significant. Two-way interactions that resulted in significant effects included treatment condition by SPP ( $F=5.7, p<.02, \mathrm{df}=1 / 593)$, type of prior drinking experience by SPP $(\mathrm{F}=7.7, p<.001$, df $=$ $2 / 593)$, and SPP by testing occasion $(F=38.2$, $p<.001, \mathrm{df}=3 / 1,799)$. Neither the four-way nor any of the three-way interactions were significant. The intraclass correlation coefficient for alcohol frequencyquantity was .02 yielding a design effect of 1.6. When corrected for the design effect, the F-ratios were 28.4 
possibility is complicated by the finding, noted in an earlier article, ${ }^{2}$ that the differential program effect on AUM was not noted among students who received the intervention in the fifth grade. As noted in this and the earlier article, however, the differential program effect on alcohol misuse among those who entered the study in sixth grade did not reach statistical significance until the final posttest, which occurred in the eighth grade for those students. Students who received the intervention in the fifth grade were in seventh grade at the final posttest, thus no eighth grade comparisons were possible. Additional longitudinal research is required to determine whether students who receive the schoolbased, social skills intervention earlier than sixth grade show the subsequent greater reductions compared with a control group in rates of increase in SPP, early experimentation with unsupervised alcohol use, and subsequent alcohol misuse.

Another avenue of research suggested by these findings is the need for conducting tests of the effectiveness of specific components of school-based, social pressure resistance skills prevention programs in reduction of SPP and alcohol misuse. In overall tests of program effectiveness, as conducted in this study, it is not possible to ascertain which aspects of the program are most effective in achieving these reductions. One set of hypotheses to be considered is that changing the adolescents' perceptions of family and other significant adult role modeling, as well as family and peer group norms and behavior with respect to AUM is important in achieving reductions in adolescent SPP and AUM. If this is the case, it would be useful to test earlier interventions conducted in the school, with peer norms as a component, as well as earlier family-based interventions. Such components might provide parents with information and skills directed at changing children's perceptions of peer norms and behavior, and with effective methods of parenting, including the setting, monitoring, and enforcing of family norms regarding AUM.

Though research exists concerning peer and family antecedents of adolescent AUM, ${ }^{17-21}$ comprehensive longitudinal tests of causal models have not been conducted. Longitudinal research needs to be conducted to simultaneously test the direct and indirect influences of peer norms and peer behavior, as well as family norms, parental norm setting, monitoring and enforcement, and parental and other significant adult role modeling behavior. This type of research would be extremely useful in providing direction for the development and testing of components of adolescent alcohol misuse prevention programs.

\section{CONCLUSION}

These results support the continued development of school-based alcohol misuse prevention programs based on providing practice in the social skills to resist "social pressure" to use and misuse alcohol. The prevention program should include components hypothe- sized to reduce SPP. Realistic information regarding peer group norms and behavior with respect to AUM may be as important as the behavioral skills to resist peer pressure. Longitudinal, developmental studies are needed to test family-based interventions that include information concerning peer norms and behavior, as well as role modeling behavior by parents and other significant adults and parenting skills that focus on norm setting, monitoring, and enforcement.

\section{References}

1. Goodstat M. School-based drug education in North America: What is wrong? What can be done. J Sch Health. 1986;56(7):278-281.

2. Dielman TE, Shope JT, Leech SL, Butchart AT. Differential effectiveness of an elementary school-based alcohol misuse prevention program. J Sch Health. 1989;59(6):255-263.

3. Dielman TE, Butchart AT, Shope JT, Campanelli PC, Caspar R. A covariance structure model test of antecedents alcohol misuse and a prevention effort. $J$ Drug Educ. 1989;19(4):337-361.

4. Dielman TE, Shope JT, Butchart AT, Campanelli PC. Prevention of adolescent alcohol misuse: An elementary school program. $J$ Pediatr Psychol. 1986;11:259-282.

5. Shope JT, Dielman TE, Butchart AT, Campanelli PC, Kloska DD. An elementary school-based alcohol misuse prevention program: A follow-up evaluation. $J$ Stud Alcohol. 1992;53:106-121.

6. Shope JT, Dielman TE, Smith L, Lorenger AT, et al. Alcohol Misuse Prevention: Curriculum guide for fifth and sixth grades. Ann Arbor, Mich: University of Michigan, Office of Educational Resources and Research; 1985.

7. Shope JT, Dielman TE. Alcohol Misuse Prevention: Curriculum guide for year two sixth grade booster sessions. Ann Arbor, Mich: University of Michigan, Office of Educational Resources and Research; 1986.

8. Campanelli PC, Dielman TE, Shope JT, Butchart AT, et al. Pretest and treatment effects in an elementary school-based alcohol misuse prevention program. Health Educ $Q$. 1989;16:113-130.

9. Campanelli PC, Dielman TE, Shope JT. Validity of adolescents self-reports of alcohol use and misuse using a bogus pipeline procedure. Adolescence. 1987;22:7-21.

10. Dielman TE, Campanelli PC, Shope JT, Butchart AT Susceptibility to peer pressure, self-esteem and health locus of control as correlates of adolescent substance abuse. Health Educ $O$. 1987;14: 207-221.

11. SPSS-X User's Guide, 3rd ed. Chicago, Ill: SPSS Inc; 1988.

12. McAlister A, Perry C, Maccoby N. Adolescent smoking: Onset and prevention. Pediatrics. 1979;63:650-658.

13. Moskowitz J, Malvin J, Shaefer G. Schaps E. An experimental evaluation of a drug education course. $J$ Drug Educ. 1984;14:9-22.

14. Murray DM, Hannan PJ. Planning for the appropriate analysis in school-based drug-use prevention studies. J Consult Clin Psychol. 1990;58:548-568.

15. Hopkins K. The unit of analysis: Group means versus individual observations. Am Educ Res $J$. 1982;19:5-18.

16. Kish L. Survey Sampling. New York, NY: Wiley; 1965.

17. Huba GJ, Bentler PM. The role of peer and adult models for drug taking at different stages of adolescence. $J$ Youth Adoles. 1980;9:449-465.

18. Brooks JS, Whiteman M, Gordon AS, Nomura C, et al. Onset of adolescent drinking: A longitudinal study of intrapersonal and interpersonal antecedents. Adv Alcohol Subst Abuse. 1986;5:91-110.

19. Gforer J. Correlation between drug use by teenagers and drug use by older family members. Am J Drug Alcohol Abuse. 1987; 13:95-108

20. Dielman TE, Butchart AT, Shope JT, Miller M. Environmental correlates of adolescent substance use and misuse: Implications for prevention programs. Int J Addict. 1991;25(7A \& 8A):857-882.

21. Dielman TE, Butchart AT, Shope JT. Structural equation model tests of patterns of family interaction, peer alcohol use, and intrapersonal predictors of adolescent alcohol use and misuse. $J$ Drug Educ. To be published. 